\title{
A classification of continua by certain cutting properties
}

by

\section{E. S. Thom as, Jr.* (Ann Arbor, Mich.)}

Introduction. By a continuum we mean a compact connected metric space. A subcontinuim of a space $X$ is a closed connected subset of $X$ and by $S(X)$ we mean the collection of subcontinua of $X$.

Let $M$ be a continuum and $U, V$ open subsets of $M$. A collection $C \subseteq S(M)$ cuts between $U$ and $V$ in $M$ provided no member of $C$ meets $U \cup V$ and every member of $S(M)$ which meets both $U$ and $V$ meets a member of $C$. A subcollection $C$ of $S(M)$ separates $M$ in case $M-\cup C$ is disconnected. Thus, in general, cutting is weaker than separating.

With each nondegenerate continuum $M$ we associate a number $n$ as follows: $n$ is the smallest cardinal $z$ such that some subcollection $C$ of $S(M)$ with cardinality $z$ cuts between a pair of open sets in $M$. We say that $M$ is of type $n$. Thus an arc is of type 1 and a simple closed curve is of type 2 .

The initial motivation for the work presented here was a conversation with F. Burton Jones in which the question arose: Is every continuum of type $n, 2 \leqslant n<s_{0}$, separated by $n$ of its subcontinua?. We show that the answer is yes (cf. Corollaries 2.2 and 3.2 below). This follows from some structure theorems for such continua. Our major results (Theorems 2.3 and 3.1) say that if $M$ is of type $n$ where $2 \leqslant n<s_{0}$ then by collapsing $n$ subcontinua of $M$ points one obtains a continuum of type $n$ consisting: of two indecomposable continua meeting at $n$ points. No neat analogues of the results for finite $n$ have been found for continua of type $s_{0}$, in fact, we give an example of a continuum $M$ of type $s_{0}$ every indecomposable subcontinuum of which is nowhere dense in $\boldsymbol{M}$.

Throughout the paper we use the notation $X^{\circ}$ and $\bar{X}$ to denote, respectively, the interior of $X$ and the closure of $X$ in $M$. Relative interior and closures will be written out in words.

1. Throughout sections 1 and 2 let $M$ denote a continuum of type $n$ where $3 \leqslant n<s_{0}$ and let $\left\{A_{1}, \ldots, A_{n}\right\} \subset S(M)$ cut between the open sets $U_{0}$ and $V_{0}$ of $M$. Let $\mathfrak{P}$ denote the collection of ordered pairs $\left(U^{\prime}, \nabla^{\prime}\right)$

* Partially supported by the National Science Foundation. 
where $U^{\prime}, V^{\prime}$ are open sets containing $U_{0}, V_{0}$ respectively, and $A_{1}, \ldots, \lambda$ cut between $U^{\prime}$ and $V^{\prime}$. Order $\mathfrak{P}$ in the natural way: $\left(U^{\prime}, V^{\prime}\right) \leqslant\left(U^{\prime \prime}, V^{\prime \prime}\right)$ provided $U^{\prime} \subseteq V^{\prime \prime}, V^{\prime} \subseteq U^{\prime \prime}$; by Zorn's lemma there is a maximal pair in $\mathfrak{P}$. In sections 1 and $2(U, V)$ denotes some fixed maximal pair.

We now list some useful facts.

LeMma 1.1. (a) If $K \in S(M)$ meets at least two of the $A_{i}$ then $K$ contains $U$ or $V$.

(b) If $K_{1}, K_{2}$ are in $S(M)$ and $K_{1}^{\circ}, K_{2}^{\circ}$ meet $U$ and $V$, respectively, then $\left(K_{1} \cup K_{2}\right)^{\circ}$ contains at least $n-1$ of the $A_{i}$.

Proof. Part (a) is obvious. To prove (b) suppose $K_{1}, K_{2} \in S^{\prime}(M)$ and $A_{1}, A_{2}$ contain points $a_{1}, a_{2}$, resp., not in $\left(K_{1} \cup K_{2}\right)^{\circ}$. Since $K_{1}, K_{2}$ do not cut between any pair of sufficiently small open neighborhoods of $a_{1}$ and $a_{2}$, there is, for each positive integer $i$, a $C_{i} \in S(M)$ such that $C_{i} \cap\left(K_{1} \cup K_{\infty}\right)=\varnothing$ and each of $\operatorname{dist}\left(a_{1}, C_{i}\right)$, dist $\left(a_{2}, C_{i}\right)$ is less than $1 / 2$. Then $C=\bigcap_{j=1}^{\infty} \overline{U\left\{C_{i} \mid i \geqslant j\right\}}$ is a subcontinuum of $M$ containing $a_{1}$ and $a_{2}$ and missing $K_{1}^{\circ}$ and $K_{2}^{\circ}$. Since, $C$ meets $A_{1}$ and $A_{2}$, it contains $U$ or $V$ (by part (a)); say $U \subset C$. Thus both of $K_{1}^{\circ}$ and $K_{2}^{\circ}$ miss $U$.

LEMMA 1.2. $U$ and $V$ are connected.

Proof. Since no subcontinuum of $M$ separates $M$, it suffices to show that $M-U$ and $M-V$ are connected. We first show that some component of $M-U$ contains all the $A_{i}$. If this is not the case, then $M-U=E+F$, separated sets, where we may assume $A_{1} \subseteq E, A_{2} \subseteq F$. We may also assume $F^{\circ} \cap V \neq \varnothing$. Since $A_{2}, \ldots, A_{n}$ do not cut between any pair of open sets in $M$, there is $K \in S(M)$ such that $K$ joins $U$ to $F^{\circ} \cap V$ and $K$ misses each $A_{i}$ which lies in $F$. There is a subcontinuum $K^{\prime}$ of $K$ which meets $U$. and $F^{\circ} \cap V$ and misses $E$. Then $K^{\prime}$ misses every $A_{i}$ but joins $U$ to $V$, a contradiction.

Thus there is a component $A$ of $M-U$ containing all the $A_{i}$. By part (a) of Lemma 1.1, $\vee \subset A$. If $A \neq M-U$, write $M-U=E \cup F$ nonvoid and separated, where $A \subseteq E$. It is easily shown that $(U \cup F, V)$ is a member of $\mathfrak{B}$; this contradicts maximality of $(U, V)$ in $\mathfrak{P}$. Thus $A=M-U$, i.e. $M-U$ is connected. Similarly $M-V$ is connected.

In the remainder of section 1 and throughout section $2, P$ and $Q$ will denote the continua $\bar{U}$ and $\bar{V}$, respectively. We remark that since $n \geqslant 3, M-(P \cup Q)$ is connected, whence $M-\overline{(M-P \cup Q)}=(P \cup Q)^{\circ}$ is connected. In particular $P \cup Q$ is connected.

Lemma 1.3. One of $P, Q$ is indecomposable.

Proof. Suppose to the contrary that each of $P$ and $Q$ is the union of two proper subcontinua: $P=P_{1} \cup P_{2}, Q=Q_{1} \cup Q_{2}$. Each of $P_{1}, Q_{1}$ has nonvoid interior relative to $M$; thus by part (b) of Lemma 1.1; $P_{1} \cup Q_{1}$

contains $n-1$, hence at least two, of the $A_{i}$. By part (a) of Lemma 1.1, $P_{1} \cup Q_{1}$ contains $U$ or $V$, say $U \subset P_{1} \cup Q_{1}$. Since $Q \cap U=\varnothing$, we have $U \subset P_{1}$ which implies that $\bar{U}=P \subseteq P_{1}$, contradicting $P_{1} \subset P$.

THEOREM 1.4. Both $P$ and $Q$ are indecomposable.

Proof. By Lemma 1.3 we may assume that $P$ is indecomposable. Before proving the theorem we establish the following auxiliary result.

(A) $Q$ is indecomposable if there is a subcollection $\left\{A_{1}^{\prime}, \ldots, A_{n}^{\prime}\right\}$ of $S(M)$ satisfying:
(i) $A_{i} \subseteq \underline{A_{i}^{\prime}}, i=1, \ldots, n$,
(ii) $A_{i}^{\prime}-P^{\circ}=\emptyset, i=1, \ldots, n$,
(iii) $U \not \subset \bigcup_{i=1}^{n} A_{i}^{\prime}$
(iv) $V \cap A_{i}^{\prime}=\varnothing, i=1, \ldots, n$.

For suppose such a collection exists and $Q$ is the union of proper subcontinua $Q_{1}$ and $Q_{2}$. Denote by $K$ the subcontinuum $\overline{M-\left(P \cup Q_{1}\right)}$ of $M$. Since $Q_{1}$ is proper in $Q, V \not \subset K$. Also $U \cap K=\emptyset$, so by (iii) and (iv) above, $K \cup A_{1}^{\prime} \cup \ldots \cup A_{n}^{\prime}$ contains neither $U$ nor $V$. By (i) and part (a) of Lemma 1.1 we conclude that $K$ meets at most one $A_{i}^{\prime}$. Hence, reordering the $A_{i}^{\prime}$ if necessary, we have: $A_{1}^{\prime} \cup \ldots \cup A_{n-1}^{\prime} \subset M-K=\left(P \cup Q_{1}\right)^{\circ} \subseteq P^{\circ} \cup$ $\cup Q_{1}$. From (ii) it follows that $Q_{1}$ meets at least two of the $A_{i}$ and since $Q_{1}$ is proper in $Q$, this contradicts part (a) of Lemma 1.1. Thus, statement (A) is proved.

To prove Theorem 1.4 we construct a family $A_{1}^{\prime}, \ldots, A_{n}^{\prime}$ having properties (i)-(iv). The construction splits into two cases.

Case 1 . All the $A_{i}$ miss $Q$. In this case $P^{\circ}$ contains at least $n-1$ of the $A_{i}$, otherwise the continuum $\overline{M-(P \cup Q)}$ meets at least two of the $A_{i}$ contradicting Lemma 1.1, part (a). Let $i$ be an integer, $1 \leqslant i \leqslant n$. If $A_{i} \subset P^{\circ}$ construct $A_{i}^{\prime}$ as follows. Pick $K \in S(M)$ meeting $U$ and $V$ and missing $\bigcup\left\{A_{j} \mid j \neq i\right\}$; thus $K \cap A_{i} \neq \emptyset$. There is a component $C$ of $K \cap P^{\circ}$ meeting $A_{i}$ and we let $A_{i}^{\prime}=A_{i} \cup \bar{C}$. Since $P$ contains at least two of the $A_{i}$, each $A_{i}^{\prime}$ constructed in the above way lies in some composant of $P$. If $A_{i}-P^{\circ} \neq \varnothing$ we merely set $A_{i}^{\prime}=A_{i}$. Properties (i), (ii) and (iv) are easy to verify for the $A_{i}^{\prime}$ we have defined, and property (iii) follows from the fact that each $A_{i}^{\prime}$ either lies in a composant of $P$ or is one of the original $A_{i}$ (hence misses $U$ ).

Case $2 . Q$ meets some $A_{i}$. We may assume $Q \cap A_{1} \neq \varnothing$. If $2 \leqslant i \leqslant n$ and $A_{i} \subset P^{\circ}$ then construct $A_{i}^{\prime}$ as follows. Let $K \in S(M)$ join $U$ to $V$ missing $\bigcup\left\{A_{j} \mid j \neq i, j \neq n\right\} \cup\left\{u_{0}\right\}$ where $u_{0}$ is some point of $U$. If $K$ misses $A_{n}$ then it must meet $A_{i}$ and we construct $A_{i}^{\prime}$ as we did in Case 1. (Here $A_{i}^{\prime}$ will lie in a composant of $P$ because $K$ does not contain $u_{0}$.) 
Suppose then that $K$ meets $A_{n}$. Now $V-K \neq \varnothing$ since $Q$ meets $A_{1}$ and $K \cap A_{1}=\emptyset$. Hence there is $L \in S(M)$ meeting $V-K$ and $U$ and missing $\bigcup\left\{A_{j} \mid j \neq i, j \neq n\right\} \cup\left\{K \cup A_{n}\right\}$. Now $L$ meets $A_{i}$ and does not contain $U$ since $K \cap U \neq \emptyset$ and $L \cap K=\emptyset$. We now define $A_{i}^{\prime}$ as in Case 1, using $L$ in place of $K$. Again $A_{i}^{\prime}$ lies in a composant of $P$. As before, if $A_{i}-P^{\circ} \neq \varnothing$ we let $A_{i}^{\prime}=A_{i}$. The $A_{i}^{\prime}$ satisfy (i)-(iv). This completes the proof of the theorem.

Before proceeding we briefly describe what happens if $M \neq P \cup Q$. For the next result we assume $M-(P \cup Q) \neq \varnothing$ and denote by $K$ the continuum $\overline{M-(P \cup Q)}$. By Lemma 1.1, part $(\mathrm{a}),(P \cup Q)^{\circ}$ contains at least $n-1$ of the $A_{i}$; say $A_{1} \cup \ldots \cup A_{n-1} \subset(P \cup Q)^{\circ}$.

The main facts about $K$ are summarized in the following theorem.

THEOREM 1.5 (a) If $K$ is of type $m$ then $m \geqslant n-1$.

(b) $K$ is irreducible about each of the sets $K \cap P$ and $K \cap Q$.

(c) If $K_{1} \in S(K)$ has nonvoid interior relative to $K$, then $K_{1}$ meets each of the sets $Q-P, P-Q$ and $A_{n}$.

Proof. (a) Suppose that $X$ and $Y$ are nonvoid sets open in $K$ and that $K_{1}, \ldots, K_{r} \in S(K)$ cut $X$ from $Y$ in $K$. Then $X-(P \cup Q)$ and $Y-(P \cup Q)$ are nonvoid open subsets of $M$ and the $r+1$ continua $K_{i}, \ldots, K_{r}$, $P \cup Q$ cut between them. Hence $r+1 \geqslant n$ and if $K$ is of type $m$ then $m+1 \geqslant n$.

(b) Suppose that $K_{1}$ were a proper subcontinuum of $K$ and $K \cap P \subset K_{1}$. (We do not exclude the possibility that $K \cap P=\emptyset$.) Then the subcon. tinua $Q$ and $K_{1}$ would separate $M$, contradicting $n \geqslant 3$.

(c) Let $K_{1} \in S(K)$ have nonvoid interior in $K$ (hence also $K_{1}^{\circ} \neq \varnothing$ ). Suppose $K_{1} \cap(Q-P)=\emptyset$ and consider the set $D=M-\left(P \cup \bar{K}-K_{1}\right)$. $Q^{\circ}$ is an open subset of $D$. It is also closed in $D$, for if $x$ is a point of $D$ which is a limit point of $Q^{\circ}$ then $x \in Q$ and $x \notin P$ so that $x \notin K_{1}$. Since also $x \notin K-K_{1}$, we have $x \notin P \cup K \cup\left(K-K_{1}\right)=P \cup K$, i.e., $x \in Q^{\circ}$. So $Q^{\circ}$ is open and closed in $D$. Since $D$ contains points not in $Q^{\circ}$ (namely, points of $K_{1}^{\circ}$ ), $D$ is disconnected. Since $\overline{K-K_{1}} \in S(M)$ (by part (a) of this theorem) there is a contradiction to $n \geqslant 3$.

Finally we show $K_{1} \cap A_{n} \neq \emptyset$. If $K_{1}^{\circ}-A_{n}=\emptyset$ we are done, so assume $K_{1}^{\circ}-A_{n} \neq \emptyset$. Since $(U, V)$ is a maximal pair, we may choose $L_{1}, L_{2} \in S(M)$ joining $K_{1}^{\circ}-A_{n}$ to $U$ and $V$ respectively and missing $A_{1}, \ldots, A_{n}$. Then $L_{1} \cup L_{2} \cup K_{1}$ meets some $A_{i}$; since $L_{1}, L_{2}$ miss all the $A_{i}, K_{1}$ must meet this $A_{j}$. Since $A_{1} \cup \ldots \cup A_{n-1}$ lies in $(P \cup Q)^{\circ}$ and hence misses $K_{1}$, we must have $j=n$ as asserted.

There are several natural questions we might ask about the way in which $A_{1}, \ldots, A_{n}$ and $K$ (if it exists) are situated in a continuum $M$ of type $n$. For example: Do some (or all) of the $A_{i}$ meet $P \cap Q$ ? Must each of $P$ and $Q$ meet some $A_{i}$ ? (i.e.: Is Case 1 in the proof of Theorem 1.4 impossible?) Is the number of components of $P \cap Q$ (or $K \cap P$ or $K \cap Q$ ) restricted by $n$ ? Does $A_{1} \cup A_{2} \cup \ldots \cup A_{n} \cup K$ separate $M$ ?

The following example shows that the answer in each case is no. Let $k \geqslant 2$ be an integer; we indicate how to construct a continuum $M$ of type 3 in which $A_{1}, A_{2}, A_{3}$ may be chosen so that:

(1) $A_{1} \cup A_{2} \subset P^{\circ}$ and $A_{3} \cap(P \cup Q)=\emptyset$.

(2) $K$ is nonvoid and each of $P \cap Q, K \cap P, K \cap Q$ has $k$ components.

(3) $\quad M-\left(A_{1} \cup A_{2} \cup A_{3} \cup K\right)$ is connected.

The continuum $M$ may be obtained as the union of three indecomposable continua $P, Q, K$ in the plane which intersect as indicated in figure 1.

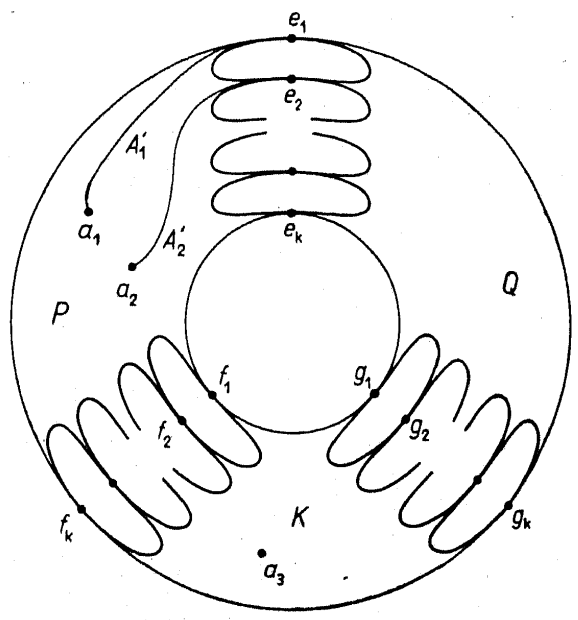

Fig.

$P, Q$ and $K$ are to be chosen so that the following conditions hold:

$$
P \cap Q=\left\{e_{1}, \ldots, e_{k}\right\} ; \quad K \cap P=\left\{f_{1}, \ldots, f_{k}\right\} ; \quad K \cap Q=\left\{g_{1}, \ldots, g_{k}\right\} ;
$$

$a_{1}$ and $a_{2}$ lie in $P-(Q \cup K)$, and $a_{3}$ is in $K-(P \cup Q)$. $A_{1}^{\prime}$ and $A_{2}^{\prime}$ lie in composants of $P ; A_{1}^{\prime}$ contains $a_{1}$ and $e_{1}$, while $A_{2}^{\prime}$ contains $a_{2}, e_{2}, \ldots, e_{k}$. Let $C_{0}$ be the composant of $P$ containing $A_{2}^{\prime}$; then $O_{0}$ contains none of $e_{1}, f_{1}, \ldots, f_{k}$. Finally, if $C$ is a composant of $P, Q$ or $K$ and $C \neq C_{0}$ then $C$ contains at most one of $e_{1}, f_{1}, \ldots, f_{k}, g_{1}, \ldots, g_{k}$.

For $i=1,2,3$ let $A_{i}=\left\{a_{i}\right\}$; then $M$ is of type 3 ; the subcontinua $A_{1}, A_{2}, A_{3}$ cut between the pair $P-\left(A_{1}^{\prime} \cup A_{2}^{\prime} \cup K\right)$ and $Q-(P \cup K)$ 
and if $(U, V)$ is a maximal pair corresponding to $A_{1}, A_{2}, A_{3}$ then (modulo a relabeling) $\bar{U}=P$ and $\bar{V}=P$. It is easy to verify $(1),(2)$ and $(3)$.

As is indicated by property (3) of this example some additional work is needed to show that a continuum of type $n$ is separated by $n$ of its subcontinua.

2. Before proving the main theorem of this section we introduce some notation and establish some basic facts.

For each point $x$ of $P$ [resp., $Q]$ let $P(x)[Q(x)]$ denote the $x$-composant of $P[Q]$. If $x \in P \cap Q$ then there is a unique integer $i$, denoted by $i(x)$, such that $P(x) \cup Q(x)$ meets $A_{i}$. (There is at least one such integer because the $A_{i}$ cut $U$ from $V$, and at most one because of Lemma 1.1, part (a).)

Observe that if $x$ and $y$ are in $P \cap Q$ and $i(x) \neq i(y)$ then $(P(x) \cup$ $\cup Q(x)) \cap(P(y) \cup Q(y))=\varnothing$, for otherwise there would be a continuum not containing $U$ or $V$ but joining $A_{i(x)}$ to $A_{i(y)}$. For $j=1, \ldots, n$, denote by $C_{j}$ the set of $x$ in $P \cap Q$ such that $i(x)=j$. As before we may assume $A_{1} \cup \ldots \cup A_{n-1} \subset(P \cup Q)^{\circ}$. It follows that each of $C_{1}, \ldots, C_{n-1}$ is nonvoid. (As the example of the preceding section shows, $O_{n}$ may be void.) In any case, the $U_{i}$ are disjoint and their union is $P \cap Q$.

THEOREM 2.1 Assume the above notation. There exist continua $K_{1}, \ldots, K_{n}$ in $M$ such that:

(a) $C_{i} \cup A_{i} \subseteq \dot{K}_{i}$ for $i=1, \ldots, n-1$;

(b) $C_{n} \cup A_{n} \cup K \subseteq K_{n}$ (where $C_{n}$ or $K$ may be void);

(c) $K_{i} \subset M-(U \cup V)$ for $i=1, \ldots, n$.

Proof. (In what follows, if $C_{n}=\emptyset$ the corresponding definition will be vacuous.) Fix $i \leqslant n$ and let $x$ be a point of $C_{i}$; it is easy to verify that exactly one of the following occurs:

(p) There is a continuum $\hat{P}(x)$ lying in $P(x)$ missing $U$ and irreducible from $x$ to $A_{i}$.

(q) There is a continuum $\hat{Q}(x)$ lying in $Q(x)$ missing $V$ and irreducible from $x$ to $A_{i}$.

Correspondingly, we define $C_{i}(P)$ and $C_{i}(Q)$ to be the set of $x$ in $C_{i}$ enjoying properties (p) or (q) respectively. Thus $C_{i}$ is the union of the disjoint sets $C_{i}(P)$ and $C_{i}(Q)$.

Let

$K_{i}(P)=A_{i} \cup \overline{\left.\langle\hat{P}(x)| x \in C_{i}(P)\right\}}$ and $K_{i}(Q)=A_{i} \cup \bigcup\left\{\overline{\left.\hat{Q}(x) \mid x \in C_{i}(Q)\right\}}\right.$ Since each $\hat{P}(\grave{x})$ misses $U$ and lies in $P, K_{i}(P)$ is a subcontinuum of $M$ missing $U \cup V$; similarly for $K_{i}(Q)$. Let $K_{i}=K_{i}(P) \cup K_{i}(Q)$ for $i<n$ and let $K_{n}=K_{n}(P) \cup K_{n}(Q) \cup A_{n} \cup K$. Properties (a), (b) and (c) follow immediately from the construction.
COROLlaRY 2.2. Each continuum of type $n, 3 \leqslant n<\kappa_{0}$, is separated by $n$ of its subcontinua. More specifically, if $K_{1}, \ldots, K_{n}$ are the continua given in Theorem 2.1 then $M-\left(K_{1} \cup \ldots \cup K_{n}\right)$ is the union of two connected open sets one containing $U$ and the other $V$.

Proof. This follows immediately from properties (a), (b) and (c) and the fact that $P \cap Q=C_{1} \cup \ldots \cup C_{n}$.

By a basic continuum of type $n\left(3 \leqslant n<\kappa_{0}\right)$ we mean a continuum $M$ of type $n$ for which the $A_{i}$ may be chosen so that each $A_{i}$ is a singleton, say $A_{i}=\left\{a_{i}\right\} ; P \cap Q=\left\{a_{i} \mid i=1, \ldots, n\right\}$ and $M=P \cup Q$. Clearly these conditions on the $A_{i}$ determine $U$ and $V$ uniquely; $U=P-Q, V=Q-P$.

Intuitively, the basic continua of type $n$ are the prototypes for arbitrary continua of type $n$. This idea is expressed more precisely in the next theorem.

THEOREM 2.3. Let $M$ be a continuum of type $n$. Then there is a basic continuum $N$ of type $n$ and a monotone continuous function $q$ mapping $M$ onto $N$.

Proof. Using the notation of this section, let $K_{1}, \ldots, K_{n}$ be the continua given in Theorem 2.1. Let $D$ denote the decomposition of $M$ whose nondegenerate elements are $K_{1}, \ldots, K_{n}$. Then $D$ is monotone and upper semi-continuous. Let $N$ denote the quotient space of $M$ modulo $D$ and $q$ the quotient map.

$N$ is a continuum and using the fact that the $K_{i}$ separate $M$ and the monotonity of $q$ it is easy to verify that $N$ is of type $n$.

Let $U^{\prime}=q(U)$ and $V^{\prime}=q(V)$; then $U^{\prime}$ and $V^{\prime}$ are open in $N$ and the points $q\left(K_{1}\right), \ldots, q\left(K_{n}\right)$ cut between (in fact, separate) these two sets in $M$. Since $N=U^{\prime} \cup V^{\prime} \cup\left(q\left(K_{1}\right) \cup \ldots \cup q\left(K_{n}\right)\right)$ the pair $\left(U^{\prime}, V^{\prime}\right)$ is maximal. Now the closures in $N$ of $U^{\prime}$ and $V^{\prime}$ are just $q(P)$ and $q(Q)$, respectively (where $M=P \cup Q \cup K$ in the usual way). Since $q$ is monotone and continuous, $q(P)$ and $q(Q)$ are indecomposable. Thus $N=q(P) \cup$ $\cup q(Q)$ is basic.

3. Let $M$ be a continuum of type 2. If $A_{1}, A_{2}$ cut between a pair of open sets then again there is a maximal pair $(U, V)$. As in section 1 one can show that $M-U, M-V, U$ and $V$ are all connected, and, as before, we let $P=\bar{U}, Q=\bar{V}$.

Since a simple closed curve is of type 2, neither $P$ nor $Q$ need be indecomposable. If $P$ and $Q$ are indecomposable; $P \cap Q=\left\{a_{1}, a_{2}\right\}$ where $A_{i}=\left\{a_{i}\right\}, i=1,2$; and $P \cup Q=M$, then we say that $M$ is a basic continuum of type 2.

In view of the wide range of possibilities for the structure of continua of type 2 the most satisfying theorem seems to be the following analogue of Theorem 2.3. We omit many details in the proof. 
ThEOREM 3.1. Let $M$ be of type 2; there is a monotone continuous image of $M$ which is either a simple closed curve or a basic continuum of type 2.

Proof. One of the following three possibilities holds:

(a) $M-(P \cup Q)$ is disconnected and nonvoid;

(b) $M-(P \cup Q)$ is connected and nonvoid;

(c) $M=P \cup Q$.

If (a) or (b) holds then there is a monotone continuous image of $M$ which is of type 2 and for which (c) holds.

To see this, suppose that (a) holds. Then $M-(P \cup Q)$ has exactly two components whose closures we denote by $H$ and $K$. Each of $H$ and $K$ meets both $P$ and $Q$. Let $D$ be the decomposition of $M$ whose nondegenerate elements are $H$ and $K$, let $\widetilde{M}$ be the quotient space of $M$ modulo $D$ and let $q$ be the quotient map.

No subcontinuum of $\tilde{M}$ cuts between a pair of open subsets of $\tilde{M}$ since $q$ is monotone and continuous. $\widetilde{M}$ is of type 2 ; indeed, let $\widetilde{A_{1}}=q(H)$, $\widetilde{A_{2}}=q(K)$; then $\widetilde{A}_{1}, \widetilde{A}_{2}$ cut between the open sets $q(U)$ and $q(V)$ (because no continuum separates $H^{0}$ from $K^{0}$ in $\left.M\right)$. If $(\widetilde{U}, \widetilde{V})$ is the corresponding maximal pair and $\widetilde{P}, \widetilde{Q}$ are the closures of $\widetilde{U}, \widetilde{V}$ in $\widetilde{M}$ then $\widetilde{P}=q(P)$ and $\widetilde{Q}=q(Q)$ and $\underset{\widetilde{P}}{\widetilde{M}} \widetilde{Q}$ is the pair of points $\widetilde{A_{1}}, \widetilde{A_{2}}$. Finally $\widetilde{M}=\widetilde{P} \cup \widetilde{Q}$, i.e., (c) holds for $\widetilde{M}$.

If (b) holds for the original continuum $M$, then a similar result is obtained using the decomposition whose nondegenerate element is $\overline{M-(P \cup Q)}$.

To sum up, we may assume at the outset that (c) holds for $M$. Using the process of the preceding section one constructs continua $K_{1}, K_{2} \in S(M)$ such that $A_{i} \subseteq K_{i}, i=1,2$, and $M-\left(K_{1} \cup K_{2}\right)=U^{\prime} \cup V^{\prime}$, disjoint open sets, with $U \subseteq U^{\prime}$ and $V \subseteq V^{\prime}$. Moreover, each of $P, Q$ is irreducible from $K_{1}$ to $K_{2}$.

Now suppose that one of $P, Q$, say $P$, admits the unit interval $[0,1]$ as a monotone continuous image via a map $h$. Let $D$ be the decomposition of $M$ whose elements are $Q$ and the point inverses $\left\{h^{-1}[r] \mid r \epsilon(0,1)\right\}$. Then $D$ is monotone and upper semi-continuous, the quotient space is a simple closed curve and we are done.

If neither $P$ nor $Q$ maps into $[0,1]$ in the above way, then by Theorem 10, section 1 , of [1], each of them contains an indecomposable subcontinuum with (relative) nonvoid interior, say $\hat{P} \subseteq P, \hat{Q} \subseteq Q$.

If $M=\hat{P} \cup \hat{Q}$ then the quotient space modulo the decomposition whose nondegenerate elements are $K_{1}$ and $K_{2}$ is a basic continuum of type 2.

If $M-(\hat{P} \cup \hat{Q})$ is nonvoid then it has either one component, whose closure is denoted by $L$, or two, whose closures we denote by $H$ and $K$.
In the first case $L$ meets exactly one of $K_{1}$ and $K_{2}$, say $L \cap K_{1} \neq \varnothing$. We then factor $M$ by the decomposition whose nondegenerate elements are $L \cup K_{1}$ and $K_{2}$. In the second case each of $H$ and $K$ meets exactly one of $K_{1}$ and $K_{2}$ and vice versa, say $H \cap K_{1} \neq \varnothing$ and $K \cap K_{2} \neq \emptyset$, and we factor by the decomposition whose nondegenerate elements are $H \cup K_{1}$ and $K \cup K_{2}$. In either case the quotient space is basic of type 2.

As in the preceding section we have:

CoRourary 3.2. Ivery continuum of type 2 is separated by a pair of its subcontinua.

4. Much of the theory developed for continua of type $n, 2 \leqslant n<\mathbf{s}_{0}$ fails to have any counterpart in the study of continua of type $\kappa_{0}$. Some results, too complicated and incomplete to give here, have been obtained by modifying the idea of a maximal pair. To illustrate the differences encountered in passing from finite cardinals to $\kappa_{0}$ we construct an example of a continuum $M$ of type $\kappa_{0}$ and a collection $\left\{a_{i} \mid i=1, \ldots\right\}$ of points of $M$. whose union $A$ separates $M$ such that each component of $M-A$ has void interior in $M$. In particular every indecomposable subcontinuum of $M$ has void interior.

The continuum $M$ is obtained by identifying pairs of points of a Cantor set of indecomposable continua.

Let $S$ denote an indecomposable subcontinuum of the plane; let $\left\{O_{i} \mid i=0,1,2, \ldots\right\}$ be a countable pairwise disjoint collection of composants of $s$; and let $a_{0}$ be a point of $C_{0}$. Let $K$ denote a Cantor set in $[0,1]$; let $E$ denote the set of endpoints of the components of $[0,1]-K$; and let $\left\{p_{1}, p_{2}, \ldots\right\}$ denote the collection of ordered pairs $E \times E$. Write $p_{i}=\left(e_{i}, f_{i}\right), i=1,2, \ldots$

Let $T=K \times S$; we think of $T$ and all the arcs constructed below as lying in Euclidean 3 -dimensional space. Let $A_{0}$ be the arc $[0,1] \times\left\{a_{0}\right\}$. Having chosen $A_{0}, A_{1}, \ldots, A_{n-1}, n \geqslant 0$, let $A_{n}$ be an arc with endpoints $a_{n}$ and $b_{n}$ such that $A_{n} \cap\left(T \cup A_{0} \cup \ldots \cup A_{n-1}\right)=\left\{a_{n}, b_{n}\right\} ; a_{n} \in\left\{e_{n}\right\} \times C_{n}$; $b_{n} \in\left\{f_{n}\right\} \times O_{n}$, and $\operatorname{dist}\left(A_{n}, A_{0}\right) \leqslant 1 / 2^{n}$.

Let $N=T \cup \bigcup_{n=0}^{\infty} A_{n}$; then $N$ is a continuum. Let $D$ denote the decomposition of: $N$ whose nondegenerate elements are the $A_{i}, i=0,1,2, \ldots$; then $D$ is monotione and upper semi-continuous. The quotient space $M$ is a continuum of type $\kappa_{0}$ and the points of $M$ corresponding to the $A_{i}$ separate $M$. It is not difficult to see that $M$ has the properties stated in the opening paragraph of this section.

In addition to the general problem of getting a structure theory (and, perhaps, finding a reasonably simple class of prototypes) for continua of type $s_{0}$ there are at least two questions which should be answered. 
Does every continuum of type $s_{0}$ contain an indecomposable subcontinuum? Is every continuum of type $s_{0}$ separated by a countable collection of its subcontinua?

\section{Reference}

[1] E. S. Thomas, Jr., Monotone decompositions of irreducible continua, Rozprawy Mat. 50 (1965).

UNIVERSITY OF CALIFORNIA, Riverside,

and

Regu par la Rédaction le 15. 11. 1965

\section{Open mappings on graphs and manifolds*}

by

\section{Richard A. Duke (Seattle, Wash.)}

1. Introduction. In [3], pp. 182 and 197, Whyburn has shown that the image of a finite graph under a (light) open mapping is again a finite graph and also that the image under a light, open mapping of a 2-manifold is a 2-manifold. The purpose of this paper is to investigate the conditions under which an open mapping $f(G)=H$ defined on a graph $G$ can be extended to a light, open mapping $F(M)=N$ defined on a 2-manifold $M$ when $G$ and $H$ are imbedded in $M$ and $N$, respectively.

In section 3 it is shown that if $f(G)=H$ is such an open mapping, then there exist imbeddings of $G$ and $H$ in some orientable 2-manifolds $M$ and $N$ respectively, and an extension of $f$ to a light, open map $F(M)=N$. The imbedding of $H$ may be taken to be any orientable, 2-cell (and hence any minimal) imbedding of $H$. Further, any light, open map $F(M)=N$ on a closed orientable 2-manifold $M$ can be obtained as an extension of such a map $f(G)=H$ on a graph $G$ minimally imbedded in $M$. In section 4 it is shown that for each positive integer $n$ there is an open mapping of a planar graph onto some graph of genus $n$, and this is used to show that the imbedding obtained for $G$ in the above result may necessarily be non-minimal.

2. Background. The term mapping will be used to denote a continuous transformation. A mapping $f(X)=Y$ is said to be open provided that every open set in $X$ maps onto a set open in $Y$. If for each $y$ in $Y$, $f^{-1}(y)$ is totally disconnected, $f$ is said to be a light mapping. The term graph will denote a finite, connected 1-complex.

If $f(G)=H$ is an open mapping on a graph $G$, then $H$ is also a graph. Further, $f$ is a light mapping and it is possible to designate certain interior points on the edges of $G$ and $H$ as additional vertices in such a way that $f$ maps each edge of $G$ topologically onto an edge in $H$. In light of these facts, we shall assume for the remainder that any open mapping on a graph is a simplicial transformation.

* This research was supported in part by a grant from the National Science Foundation. 